\title{
A new therapy (MP29-02*) effectively controls nasal symptoms of seasonal allergic rhinitis irrespective of severity
}

\author{
Glenis Scadding ${ }^{1 *}$, David Price ${ }^{2}$, Jean Bousquet ${ }^{3}$, Peter Hellings ${ }^{4}$, Wytske Fokkens ${ }^{5}$, Ullrich Munzel ${ }^{6}$, Claus Bachert ${ }^{7}$ \\ From 9th Symposium of Experimental Rhinology and Immunology of the Nose (SERIN 2013) \\ Leuven, Belgium. 21-23 March 2013
}

\section{Background}

It is important to show efficacy in allergic rhinitis (AR) patients regardless of symptom severity since most AR patients presenting to a doctor have moderate-to-severe disease.

\section{Objective}

To assess the efficacy of MP29-02* (a novel intranasal formulation of azelastine hydrochloride [AZE] and fluticasone propionate [FP]) compared to AZE, FP or placebo nasal sprays in seasonal AR (SAR) patients according to severity.

\section{Methods}

610 patients ( $>=12$ years old) with moderate-to-severe SAR were randomized into a double-blind, placebo-controlled, 14-day, parallel-group trial to receive MP29-02\%, commercially-available AZE or FP nasal sprays or placebo (all given as 1 spray/nostril bid; total daily dose: $548 \mu \mathrm{g}$ AZE; $200 \mu \mathrm{g}$ $\mathrm{FP}])$. The primary efficacy variable was change from baseline in rTNSS $(\mathrm{AM}+\mathrm{PM})$. This primary endpoint was assessed post-hoc according to symptom severity. All patients had moderate-to-severe disease. Patients were categorized into two severity groups according to their median baseline rTNSS. Those with a baseline rTNSS > 18.9 points were defined as more severe and those with a baseline $\mathrm{rTNSS}<=18.9$ points were defined as less severe.

\section{Results}

MP29-02* was significantly superior to either FP or AZE in alleviating patients' rTNSS regardless of disease severity. For those patients with less severe disease $(<=18.9)$ MP29$02^{*}$ reduced the rTNSS from baseline by -4.68 compared

\footnotetext{
${ }^{1}$ The Royal National Throat, Nose and Ear Hospital, London, UK Full list of author information is available at the end of the article
}

to -3.21 for FP (Diff: -1.46 ; $95 \%$ CI $-2.68,-0.25 ; \mathrm{p}=0.0188$ ), -2.41 for AZE (Diff: -2.26 ; 95\% CI $-3.42,-1.10$; $\mathrm{p}=0.0002$ ) and -1.16 for placebo (Diff: -3.51 ; 95\% CI: $-4.78,-2.24$; $\mathrm{p}<0.0001$ ), corresponding to a relative treatment difference of $42 \%$ vs FP and $64 \%$ vs AZE. Patients with more severe disease $(>18.9)$ experienced a -6.24 point reduction in their rTNSS with MP29-02*, significantly more than -4.73 with FP (Diff: -1.52 ; 95\% CI -2.99, -0.04; $\mathrm{p}=0.0436$ ), -4.11 with AZE (Diff: $-2.13 ; 95 \% \mathrm{CI}:-3.55,-0.71 ; \mathrm{p}=0.0035)$ and -3.18 with placebo (Diff -3.06; 95\% CI -4.34, -1.77; $\mathrm{p}<0.0001)$. For these more severe patients, the relative treatment effect was $49 \%$ to FP and $70 \%$ to AZE.

\section{Conclusion}

MP29-02* provided benefits for all patients, offering significantly greater relief from nasal symptoms compared to two firstline therapies regardless of disease severity and is the drug of choice for the treatment of AR.

"Dymista

\section{Author details \\ ${ }^{1}$ The Royal National Throat, Nose and Ear Hospital, London, UK. ${ }^{2}$ University of Aberdeen, Dept of General Practice \& Primary Care, Aberdeen, UK. ${ }^{3}$ Hopital Arnaud de Villeneue University Hospital, Montpellier, France. ${ }^{4}$ University Hospitals Leuven, Dept of Otorhinolaryngology, Head and Neck Surgery, Leuven, Belgium. ${ }^{5}$ Academic Medical Center, Dept of Otorhinolaryngology, Amsterdam, the Netherlands. ${ }^{6}$ Meda Pharma, Biostatistics \& Market Access, Bad Homburg, Germany. ${ }^{7}$ Ghent University Hospital, Dept of Oto-rhinolaryngology, Ghent, Belgium.}

Published: 16 July 2013

doi:10.1186/2045-7022-3-S2-016

Cite this article as: Scadding et al:: A new therapy (MP29-02*) effectively controls nasal symptoms of seasonal allergic rhinitis irrespective of severity. Clinical and Translational Allergy 2013 3(Suppl 2):016.

\section{()

(c) 2013 Scadding et al; licensee BioMed Central Ltd. This is an Open Access article distributed under the terms of the Creative Commons Attribution License (http://creativecommons.org/licenses/by/2.0), which permits unrestricted use, distribution, and reproduction in any medium, provided the original work is properly cited. 